\title{
Modeling heat dominated electric breakdown in air, with adaptivity to electron or ion time scales
}

\author{
A Agnihotri ${ }^{1}$, W Hundsdorfer ${ }^{1,2}$ and $\mathbf{U}$ Ebert $^{1,3}$ \\ ${ }^{1}$ Centrum Wiskunde \& Informatica (CWI), PO Box 94079, 1090 GB Amsterdam, The Netherlands \\ ${ }^{2}$ Institute for Mathematics, Astrophysics and Particle Physics (IMAPP), Faculty of Science, HEF 79, \\ Radboud University, PO Box 9010, 6500 GL Nijmegen, The Netherlands \\ ${ }^{3}$ Department of Applied Physics, Eindhoven University of Technology, PO Box 513, 5600 MB Eindhoven, \\ The Netherlands
}

E-mail: A.Agnihotri@cwi.nl

Received 20 March 2017, revised 4 August 2017

Accepted for publication 10 August 2017

Published 30 August 2017

\begin{abstract}
We model heat dominated electrical breakdown in air in a short planar gap. We couple the discharge dynamics in fluid approximation with the hydrodynamic motion of the air heated by the discharge. To be computationally efficient, we derive a reduced model on the ion time scale, and we switch between the full model on the electron time scale and the reduced model. We observe an ion pulse reaching the cathode, releasing electrons by secondary emission, and these electrons create another ion pulse. These cycles of ion pulses might lead to electrical breakdown. This breakdown is driven by Ohmic heating, thermal shocks and induced pressure waves, rather than by the streamer mechanism of local field enhancement at the streamer tip.
\end{abstract}

Keywords: gas heating, plasma discharge, model reduction

\section{Introduction}

Gas heating in electrical discharges has a long research history. It has been studied in the context of fast gas heating [1-5], plasma-assisted combustion [6], atmospheric lightning [7] etc. On the one hand, increased temperature can change the chemical reaction rates in the discharge plasma. On the other hand, the thermal expansion of the gas can change the gas density and hence the reduced electric field in the interelectrode gap. Both mechanisms can sustain spark formation $[8,9]$, and gas heating can also create turbulent flows [10]. In the present work, we concentrate on the effect of gas heating and expansion.

So far the majority of simulation studies pertaining to gas heating in atmospheric pressure discharges have been performed in a OD or 1D spatial configuration in which the structure of discharge is rather homogeneous. Due to the large difference in timescales of gas movement and electron dynamics, solving the two systems simultaneously becomes computationally very expensive. Recently, there has been progress in extending models to $2 \mathrm{D}$ (which is meant to mean
3D with cylindrical symmetry), which brings them closer to experiments and observations as the structure of a spark discharge is usually filamentary. Komuro and Ono [11] studied the mechanism of fast gas heating and the effects of humidity in atmospheric pressure streamer discharges. They solved the coupled system of electric discharge and gas simultaneously up to time $t=3 \mu \mathrm{s}$. Tholin and Bourdon [12] simulated the hydrodynamic expansion following a nanosecond pulsed discharge in air at STP. During the short pulse they approximated the gas density as constant, hence they did not need to solve the coupled system of electric discharge and gas simultaneously. Kacem et al [13] simulated the expansion of thermal shock and pressure waves induced by streamer dynamics in positive DC corona discharges. They also do not solve the full coupled system of electric discharge and gas dynamics. However, they do remark that direct coupling of the gas dynamics with the streamer simulation should be undertaken in order to better understand the effect of temperature increase and of the gas expansion on the development of successive discharges and on the electrical relaxation. 
In the current paper, we simulate electrical breakdown in a short planar gap in $2 \mathrm{D}$ ( $r-z$ coordinates) with secondary electron emission where Ohmic heating promotes ionization growth, rather than the space charge dominated streamer breakdown mechanism. Initially only an avalanche without (streamer-type) space charge effects might have developed in a short undervolted gap, but the deposited Ohmic heat might eventually lead to electric breakdown. This is challenging to simulate as in the initial stage the electron dynamics has to be followed, and later on the much slower ion dynamics has to be resolved, and both have to be coupled to the hydrodynamics of the medium. We present a model on the time scale of ion motion that is a reduced version of the full discharge dynamics, and with adaptivity in time, i.e. we switch between dynamical calculations on electron and ion time scale as needed. In the present work, this is done manually, but we also discuss possible numerical switching criteria. The reduced model on the ion time scale was introduced in earlier work of Sijacic $[14,15]$ where it was applied to study the transition from Townsend to glow discharge.

We initiate the discharge closely below breakdown conditions, i.e., when the effective Townsend ionization coefficient $\alpha_{\text {eff }}$ is negative. As $\alpha_{\text {eff }}$ is a function of the reduced electric field, i.e., of electric field over gas density $E / N$, gas heating and subsequent expansion causes $\alpha_{\text {eff }}$ to grow, and might make it positive eventually. But that is not sufficient on time scales considerably longer than the time the ions need to cross the gap. On that time scale secondary emission from the cathode needs to sustain the discharge, and in a planar configuration with constant field and gas density, the discharge will grow further if

$$
\gamma\left(\mathrm{e}^{\alpha_{\text {eff }} d}-1\right)>1 \text {. }
$$

Here $d$ is the distance between the electrodes and $\gamma$ is the secondary electron emission coefficient which is the ratio of the number of emitted electrons over the number of ions impacting the cathode.

In our previous work [16], we developed and employed a similar 2D cylindrically symmetric model to study streamerless spark formation in supercritical $\mathrm{N}_{2}$. A limitation of that model was that it computed the relevant physical quantities on the ion timescale only, not resolving the time scale of the electron motion. Therefore we missed some initial heating effects, as we discuss here in section 3 .

The paper is organized as follows. In section 2, we introduce the mathematical model and notation for our coupled system of the electric discharge and the gas. In section 3, we discuss the reduction of the full discharge model to a model on the ion time scale. We also compare results of the full model of the electrical discharge and of the reduced model in 1D, and we point out when the reduced model can be used to speed up simulations. In section 4 , we present and discuss the results of the $2 \mathrm{D}$ simulation of the electric discharge in plane-to-plane geometry in air at standard temperature and pressure. Finally, in section 5 we summarize our findings and present an outlook.

\section{Model formulation}

We study how a pulsed electric discharge is coupled to the dynamics of the background gas due to the deposited Ohmic heat and thermal expansion. Therefore the model is composed of two components, one for the dynamics of electrons and ions in the electric discharge and one for the dynamics of the background gas.

\subsection{Model for the electric discharge dynamics}

To describe the discharge dynamics, we adopt the classical first-order drift-diffusion-reaction model [17] in local field approximation for electrons and positive and negative ions, coupled with Poisson's equation to self-consistently compute the electric field:

$$
\begin{aligned}
& \partial_{t} n_{e}+\nabla \cdot \mathbf{j}_{e}=S_{e}, \\
& \mathbf{j}_{e}=-\mu_{e} \mathbf{E} n_{e}-D_{e} \nabla n_{e}+\mathbf{v} n_{e}, \\
& \partial_{t} n_{i}+\nabla \cdot \mathbf{j}_{i}=S_{i}, \\
& \mathbf{j}_{i}=\sigma_{i} \mu_{i} \mathbf{E} n_{i}-D_{i} \nabla n_{i}+\mathbf{v} n_{i} \\
& \times \text { for ion species } i=1, \ldots \text {, } \\
& \nabla^{2} \phi=-\frac{e}{\epsilon_{0}}\left(-n_{e}+\sum_{i} \sigma_{i} n_{i}\right), \quad \mathbf{E}=-\nabla \phi .
\end{aligned}
$$

Here the lower index $e$ labels the electrons and $i=1, \ldots$ the different types of positive and negative ions. Further, $n_{e, i}$ is the number density of the charged species, $\mathbf{j}_{e, i}$ their particle current density, $\mu_{e, i}$ and $D_{e, i}$ their mobility and diffusion coefficients, and $\sigma_{i}= \pm 1$ their polarity. (Note that the electric current density carried by particle type $i$ is $e \sigma_{i} \mathbf{j}_{i}$.) $\phi$ denotes the electric potential, $\mathbf{E}$ denotes the electric field. $e$ is the elementary charge and $\epsilon_{0}$ denotes the permitivitty of vacuum. Finally, $\mathbf{v}$ is the velocity of the neutral gas through which the charged particles are moving; as the degree of ionization of the gas molecules stays below $10^{-8}$ in the presented simulations until the final stages, the neutral gas molecules provide the rest frame for the motion of the charged particles.

$S_{i}$ is the source term for the ion species with label $i$. For electrons in attaching gases like air at standard temperature and pressure we have

$$
S_{e}=(\alpha-\eta) \mu_{e} E n_{e}=\alpha_{\mathrm{eff}} \mu_{e} E n_{e},
$$

where $\alpha$ accounts for the electron impact ionization and $\eta$ for attachment, and $\alpha_{\mathrm{eff}}=\alpha-\eta$ is the effective Townsend ionization coefficient. Here, recombination or dissociative attachment or detachment are neglected to keep the notation simple, but they can easily be introduced for different gases. Similarly the method of time adaptivity introduced in the next section is not limited to the local field approximation or to the drift-diffusion-reaction model.

Finally, the discharge couples to the gas dynamics through the gas velocity $\mathbf{v}$, and because transport and reaction coefficients $\mu_{i}, D_{i}$ and $\alpha$ depend on the reduced electric field $E / N$. Here, $N$ is the number density of neutral gas molecules; it is related to the mass density $\rho$ as $\rho=m_{w} N$, where $m_{w}$ is the mass of the gas molecule. 


\subsection{Boundary conditions for the discharge}

We assume a short discharge gap between planar electrodes. To sustain the discharge, we include secondary emission of electrons from the cathode on impact of positive ions. This is implemented as a Dirichlet boundary condition for the electron density $n_{e}$

$$
\mu_{e} n_{e}=\gamma \sum_{j} \mu_{j} n_{j} \text { on the cathode. }
$$

Here $n_{j}$ is the number density of the positive ion species $j$ reaching the cathode. Furthermore, we assume that the density of positive ions vanishes on the anode and has a homogeneous Neumann boundary condition on the cathode

$$
\begin{aligned}
n_{j} & =0 \text { on the anode, } \\
\partial_{n} n_{j} & =0 \text { on the cathode, }
\end{aligned}
$$

where $\partial_{n}$ denotes the spatial derivative normal to the electrode surface. For negative ions, the role of anode and cathode are exchanged.

We remark that when the full model of electric discharge is used for computation of electron density, two boundary conditions are required. For that, we implement a homogeneous Neumann boundary condition on electron density on the anode

$$
\partial_{n} n_{e}=0 \text { on the anode. }
$$

At the lateral boundaries, we implement homogenous Neumann boundary condition for the charge densities,

$$
\left.\partial_{r} n_{e, i}\right|_{r=0}=\left.\partial_{r} n_{e, i}\right|_{r=L_{r}}=0 .
$$

The lateral boundary $\left(r=L_{r}\right)$ of the computational domain extends far from the discharge axis $(r=0)$ so that the boundary effects do not interfere with the dynamics and give rise to unphysical solutions.

\subsection{Model for the gas dynamics}

To model the gas dynamics, we adopt the compressible Euler equations without viscosity. The relevant equations for the conservation of mass, momentum and energy are in cylindrical coordinates

$$
\begin{gathered}
\frac{\partial \rho}{\partial t}+\nabla \cdot(\rho \mathbf{v})=0 \\
\frac{\partial\left(\rho v_{r}\right)}{\partial t}+\nabla \cdot\left(\rho v_{r} \mathbf{v}\right)=-\frac{\partial p}{\partial r} \\
\frac{\partial\left(\rho v_{z}\right)}{\partial t}+\nabla \cdot\left(\rho v_{z} \mathbf{v}\right)=-\frac{\partial p}{\partial z} \\
\frac{\partial \xi}{\partial t}+\nabla \cdot(\mathbf{v}(\xi+p))=S_{\xi}
\end{gathered}
$$

with $\mathbf{v}=\left(v_{r}, v_{z}\right)$ and $\nabla=\left(\partial_{r}, \partial_{z}\right)$. Here $\rho$ is the gas mass density, $\mathbf{v}$ the gas velocity, $p$ is gas pressure, and

$$
\xi=\rho \epsilon+\frac{1}{2} \rho v^{2}
$$

is the total energy per unit volume. $\epsilon$ is the internal energy per unit mass; it is related to pressure through the ideal gas law

$$
p=\rho \epsilon(\Gamma-1),
$$

where $\Gamma$ is the heat capacity ratio for the background gas whose value we assume to be constant and equal to 1.4 for air. We remark that the heat capacity will increase at the high temperatures reached at the final stages of the presented simulations, but this effect is not taken into account here.

We note that in principle the electron and ion drag force would need to be included in the momentum balance of equations $(7 b)$ and $(7 c)$, but we neglect it as ionization density in the present simulations is too low.

$S_{\xi}$ is the energy source term that models gas heating. It is modeled as follows: the electrical power density deposited by the discharge is

$$
P=e \mathbf{E} \cdot\left(-\mathbf{j}_{e}+\sum_{i} \sigma_{i} \mathbf{j}_{i}\right),
$$

where $\mathbf{j}_{i, e}$ is the particle current density of the charged species with label $i$ or of the electrons $e$. We assume that a fraction $\eta$ of the power density $P$ is converted immediately (on a nanosecond time scale) into heat $\xi[3,12]$, and the remaining $(1-\eta)$ of $P$ is first stored in vibrational states of the molecules in an energy density reservoir $\epsilon_{v}$ and released as heat with a relaxation time $\tau$. This process is modeled as

$$
\begin{gathered}
\frac{\partial \epsilon_{v}}{\partial t}=(1-\eta) P-\frac{\epsilon_{v}}{\tau}, \\
S_{\xi}=\eta P+\frac{\epsilon_{v}}{\tau} .
\end{gathered}
$$

Equation (9a) for $\epsilon_{v}$ can be integrated which leads to the final expression for $S_{\xi}$

$$
\begin{aligned}
S_{\xi}(\mathbf{r}, t)= & \eta P(\mathbf{r}, t)+(1-\eta) \\
& \times \int_{-\infty}^{t} P\left(\mathbf{r}, t^{\prime}\right) \mathrm{e}^{-\left(t-t^{\prime}\right) / \tau} \frac{\mathrm{d} t^{\prime}}{\tau} .
\end{aligned}
$$

Throughout the simulations presented here, we use $\eta=0.15$ and $\tau=20 \mu \mathrm{s}$. The values of these parameters are discussed in section 4.2.1.

\subsection{Boundary conditions for the fluid equations}

For the fluid equations describing gas flow, we implement the following boundary conditions on the electrodes $(z=0$ and $\left.z=L_{z}\right)$

$$
\begin{aligned}
& v_{z}=0, \\
& \partial_{z} p=0 .
\end{aligned}
$$

On the outer radial boundary $\left(r=L_{r}\right)$ of the computational domain, we implement an outflow boundary condition. These are not physical boundary conditions, but they are implemented for computation. The details of the implementation are discussed in appendix A.3. We remark that for the time simulated in this work, the shockwaves traveling in the radially outward direction do not reach the outer boundary. 


\section{The reduced model on the ion time scale and time adaptivity to electron or ion dynamics}

\subsection{Motivation and problem statement}

At the beginning of a pulsed discharge in a short gap, free electrons might deposit energy in the gas while drifting through the system. This is the avalanche and possibly the streamer phase, dominated by electron dynamics while the ions hardly move. At later stages, the dynamics might be dominated by the ions that move much more slowly due to their higher mass; also the heat driven expansion of the carrier gas evolves on a much longer time scale than the electron dynamics. During this stage, the electrons might form an approximately stationary distribution determined by the local electric field and gas density. But it is also possible-as we will see below-that one needs to switch back to the time scale of electron motion.

Solving the long time evolution completely on the electron time scale requires very long computation times. Therefore we here develop a method that is adaptive in time, switching between the time scales of electron or ion dynamics. In section 3.2 we extend the method of adiabatic decoupling $[14,15]$ to discharges with multiple ion species, thus deriving a reduced model on the time scale of ion motion. Then we test numerically in section 3.3 when the reduced model on the ion time scale is an appropriate approximation of the full dynamics. Finally in section 3.4, we briefly discuss criteria for switching between the full and the reduced model.

\subsection{Derivation of the reduced model on the ion time scale}

The discharge model introduced in the last section accounts for the electron time scale. However, eventually the discharge dynamics might be dominated by the ion dynamics while the much faster electrons can approach an approximately stationary distribution around the ions. And when the same number of electrons and ions are created locally, but the electrons move much faster, the space charge density is dominated by the ions. This is even more true when electron loss by attachment is included.

The derivation of an appropriate model can be formalized by methods for differential equations such as rescaling and singular perturbation theory. These methods identify which terms in an equation balance each other to form the solution, and which terms can be neglected. This balance of terms can change depending on conditions, both in space and in time. We introduced the method in $[14,15]$ under the name 'adiabatic decoupling' as it was called in early quantum mechanics to study electron dynamics in atoms and molecules where the nuclei are much heavier and slower than the electrons. In $[14,15]$, the discharge model on the ion time scale was derived for only one ion species, and assuming that mobilities and diffusion coefficients are constant. Here we generalize the derivation to several ion species and to transport coefficients depending on the local reduced field $E / N$ or other local variables.
As the basic parameter for the rescaling, we choose mobilities $\mu_{e}^{0}$ and $\mu_{1}^{0}$ of the electrons and of the dominant positive ion species for some characteristic, but arbitrary value of the reduced electric field $E / N$. Their ratio determines the small parameter

$$
\mu=\frac{\mu_{1}^{0}}{\mu_{e}^{0}} \ll 1 .
$$

Mobilities and diffusion coefficients of the ions are now rewritten as

$$
\frac{\mu_{i}(E / N)}{\mu_{e}^{0}}=\mu f_{i}(E / N), \frac{D_{i}(E / N)}{D_{e}^{0}}=\mu g_{i}(E / N),
$$

where the functions $f_{i}$ and $g_{i}$ are of order unity, and where $D_{e}^{0}$ is the electron diffusion coefficient at the same characteristic electric field as $\mu_{e}^{0}$. This notation makes explicit which terms in the equations are of order $\mu \ll 1$ or of order unity. Furthermore, we assume the gas velocity $\mathbf{v}$ to be of the order of a typical ion drift velocity or smaller, i.e.

$$
\mathbf{v}=\mu \tilde{\mathbf{v}}, \quad|\tilde{\mathbf{v}}| \leqslant \mathcal{O}\left(\mu_{1}|\mathbf{E}|\right) .
$$

Now when these relations are inserted into the discharge model $(2 a)-(2 c)$ and when the limit $\mu \rightarrow 0$ is taken, all ion currents and the gas velocity vanish, and one recovers the well known avalanche and streamer discharge model that traces the electron motion and neglects the ion motion:

$$
\begin{gathered}
\partial_{t} n_{e}+\nabla \cdot\left(-\mu_{e} \mathbf{E} n_{e}-D_{e} \nabla n_{e}\right)=S_{e}, \\
\partial_{t} n_{i}=S_{i} \text { for ion species } i=1, \ldots \\
\nabla^{2} \phi=-\frac{e}{\epsilon_{0}}\left(-n_{e}+\sum_{i} \sigma_{i} n_{i}\right), \quad \mathbf{E}=-\nabla \phi .
\end{gathered}
$$

But in a singularly perturbed set of differential equations it happens typically, that also another scaling can be chosen. When we focus on the situation where electrons and ions are created in equal rates in time, but move with different velocities, their current densities should be similar: $\mu_{e} n_{e}=\mathcal{O}\left(\mu_{1} n_{1}\right)$. This suggests to scale the electron density as

$$
\tilde{n}_{e}=\frac{n_{e}}{\mu}
$$

as $\tilde{n}_{e}$ and $n_{i}$ are of the same order of magnitude.

Furthermore, the time is rescaled to focus on the ion motion:

$$
\tilde{t}=\mu t
$$

Substituting these identities into the discharge model and using (14), we find

$$
\begin{aligned}
& \mu \frac{\partial \tilde{n}_{e}}{\partial \tilde{t}}+\nabla \cdot\left(-\mu_{e} \mathbf{E} \tilde{n}_{e}-D_{e} \nabla \tilde{n}_{e}+\mu \tilde{\mathbf{v}} \tilde{n}_{e}\right) \\
& \quad=(\alpha-\eta) \mu_{e} E \tilde{n}_{e} \\
& \quad \frac{\partial n_{1}}{\partial \tilde{t}}+\nabla \cdot\left(\mu_{e}^{0} f_{1} \mathbf{E} n_{1}-D_{e}^{0} g_{1} \nabla n_{1}+\tilde{\mathbf{v}} n_{1}\right) \\
& \quad=\alpha \mu_{e} E \tilde{n}_{e}
\end{aligned}
$$




$$
\begin{gathered}
\frac{\partial n_{i}}{\partial \tilde{t}}+\nabla \cdot\left(\sigma_{i} \mu_{e}^{0} f_{i} \mathbf{E} n_{i}-D_{e}^{0} g_{i} \nabla n_{i}+\tilde{\mathbf{v}} n_{i}\right) \\
=\tilde{S}_{i}, \tilde{S}_{i}=S_{i} / \mu, \quad \text { for ions } i=2, \ldots, \\
\nabla^{2} \phi=-\frac{e}{\epsilon_{0}}\left(-\mu \tilde{n}_{e}+\sum_{\text {ions } i} \sigma_{i} n_{i}\right), \quad \mathbf{E}=-\nabla \phi .
\end{gathered}
$$

Now the small parameter $\mu$ again appears explicitly as a linear coefficient of $\partial_{\tilde{t}} \tilde{n}_{e}$, of $\tilde{\mathbf{v}} \tilde{n}_{e}$ and of $\tilde{n}_{e}$ in the Poisson equation. Here $\mu$ acts as a singular perturbation as it multiplies the time derivative $\partial_{\tilde{t}} \tilde{n}_{e}$. Letting $\mu \rightarrow 0$ defines the model on the ion time scale. After the rescaling is undone, the discharge model on the ion time scale is finally

$$
\begin{gathered}
\nabla \cdot\left(-\mu_{e} \mathbf{E} n_{e}-D_{e} \nabla n_{e}\right)=(\alpha-\eta) \mu_{e} E n_{e} \\
\partial_{t} n_{1}+\nabla \cdot\left(\mu_{1} \mathbf{E} n_{1}-D_{1} \nabla n_{1}+\mathbf{v} n_{1}\right)=\alpha \mu_{e} E n_{e} \\
\partial_{t} n_{i}+\nabla \cdot\left(\sigma_{i} \mu_{i} \mathbf{E} n_{i}-D_{i} \nabla n_{i}+\mathbf{v} n_{i}\right) \\
=S_{i} \text { for ions } i=2, \ldots, \\
\nabla^{2} \phi=-\frac{e}{\epsilon_{0}} \sum_{\text {ions } i} \sigma_{i} n_{i}, \quad \mathbf{E}=-\nabla \phi
\end{gathered}
$$

In contrast to the original model, the time derivative of the electron density and its coupling to the gas motion has disappeared, and the electron density is also eliminated from the Poisson equation. While this is the mathematical derivation of this limit, we discussed the physical justification for the approximation at the beginning of this section.

\subsection{Numerical test of the reduced model on the ion time scale}

We now have derived two different limits of $\mu \rightarrow 0$ for the discharge model, one in equations $(15 a)-(15 c)$ on the electron time scale, and one in equations $(19 a)-(19 d)$ on the ion time scale. In analytical approaches, such equations can be linked through asymptotic matching. In section 4 , we will use these different models to perform numerical time integration that is adaptive to the state of the dynamics.

However, here we will first test whether and when the reduced model on the ion time scale $(19 a)-(19 d)$ approximates the full reaction drift diffusion model $(2 a)-(2 c)$ well.

3.3.1. Simulation conditions. In order to run simulations of the full model on the electron time scale up to $2 \mu$ s within reasonable computing times, we perform simulations in one spatial dimension. We model $\mathrm{N}_{2}$ at standard temperature and pressure with two charged species, namely electrons and $\mathrm{N}_{2}^{+}$ ions. The values of transport and reaction coefficients are taken from Montijn et al [18]. We neglect electron diffusion in the reduced model. The gas gap between two planar electrodes has a length of $L=0.46 \mathrm{~mm}$, and we assume secondary emission from the cathode $(4 a)$ with $\gamma=0.07$. The electric potential difference between the electrodes is fixed in such a manner that the average electric field is $17 \mathrm{kV} \mathrm{cm}^{-1}$, i.e., well below the breakdown value. The initial condition is a rectangular neutral seed with

$$
n_{e}=n_{+}=4.8 \times 10^{8} \mathrm{~cm}^{-3} \text { for } 0.5 L \leqslant x \leqslant 0.7 L .
$$

The full model and the model on the ion time scale are spatially discretized using the schemes described in the appendix. For the spatial grid we choose 800 points which corresponds to a spatial resolution of $0.57 \mu \mathrm{m}$. For timestepping we use the explicit trapezoidal rule for both models. We verified that further refinement of the grid did not alter the results. Therefore numerical errors are insignificant in the presented results.

3.3.2. Comparison of results. Figure 1 shows the spatial profiles of the electron number density $n_{e}(x, t)$, of the ion number density $n_{+}(x, t)$, and of the deposited energy density $\int_{0}^{t} P(x, \tau) \mathrm{d} \tau$ (with the power density $P$ of equation (8)) for different time steps. The blue lines indicate results of the full drift-diffusion-reaction model, and the red dotted line of the reduced model.

As the hydrodynamics of the gas are not followed here, the deposited energy density is determined by the discharge evolution, but does not couple back onto the discharge dynamics. The energy is included in our plots, because it shows characteristic differences between the models, that will be important for the solution of the full problem.

Let us start with discussing the full model indicated in blue.

- Initially at time $t=0$ electron and ion density are identical and no energy is deposited yet. Up to time $t \approx 7 \mathrm{~ns}$, the electrons drift out of the system while the ions do not move much. The electrons deposit energy along their drift trajectory as indicated in the right column. They also create additional electrons and ions by impact ionization along their path, but at such a low density that they are not visible on the plotted scale (except at the very last time step of $2 \mu \mathrm{s}$ ).

- During the next larger time steps from 100 to $500 \mathrm{~ns}$, the ions drift to the right while the electron density is negligible. After time $t=500 \mathrm{~ns}$ the ions hit the cathode and generate electrons by secondary emission. Therefore at time $t=750 \mathrm{~ns}$ while the ions keep arriving at the cathode, the electron density has increased to a value of the order of $10^{5} \mathrm{~cm}^{-3}$. It decreases again after $t=1 \mu \mathrm{s}$ when the ions have left the system. Note that the total energy density deposited after the primary electron and ion packages have left the system is constant in space. This is correct and can serve as a consistency check, as the same number of electrons and ions have moved over each point in space, and as the electric field is constant.

- At the last time step $t=2 \mu \mathrm{s}$, two plateaus of ion density can be seen when the plot scale is changed from $5 \times 10^{8} / \mathrm{cm}^{3}$ to $2 \times 10^{5} / \mathrm{cm}^{3}$. As the ions move through the whole length of the system in about $2 \mu \mathrm{s}$, the higher plateau of ion density near the right boundary must have been created while the initial electron pulse moved out of the system up to time $t=10 \mathrm{~ns}$. The second lower 

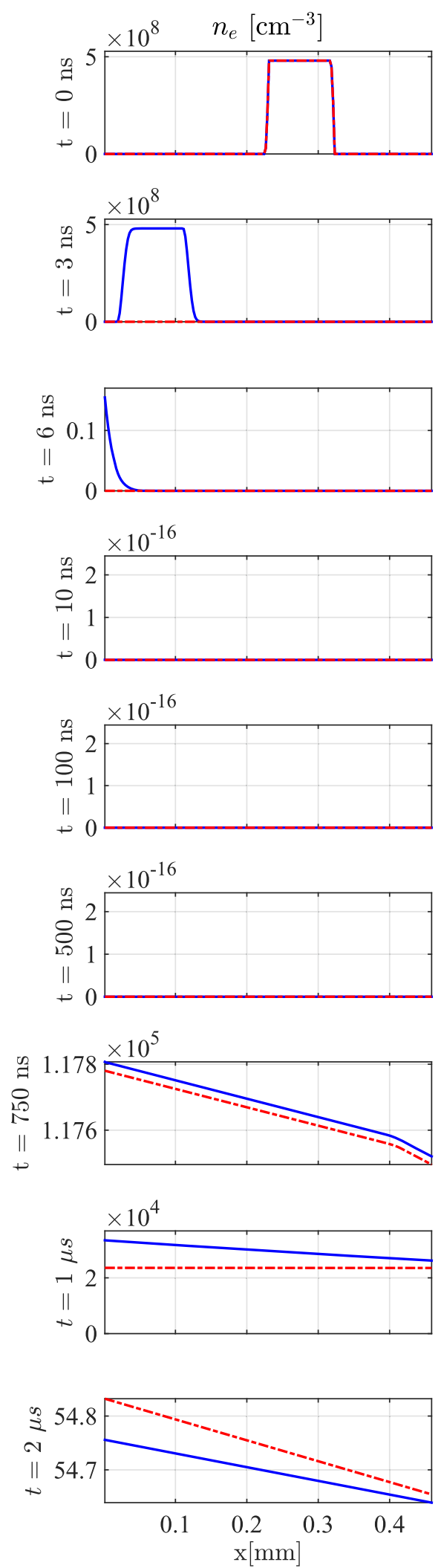
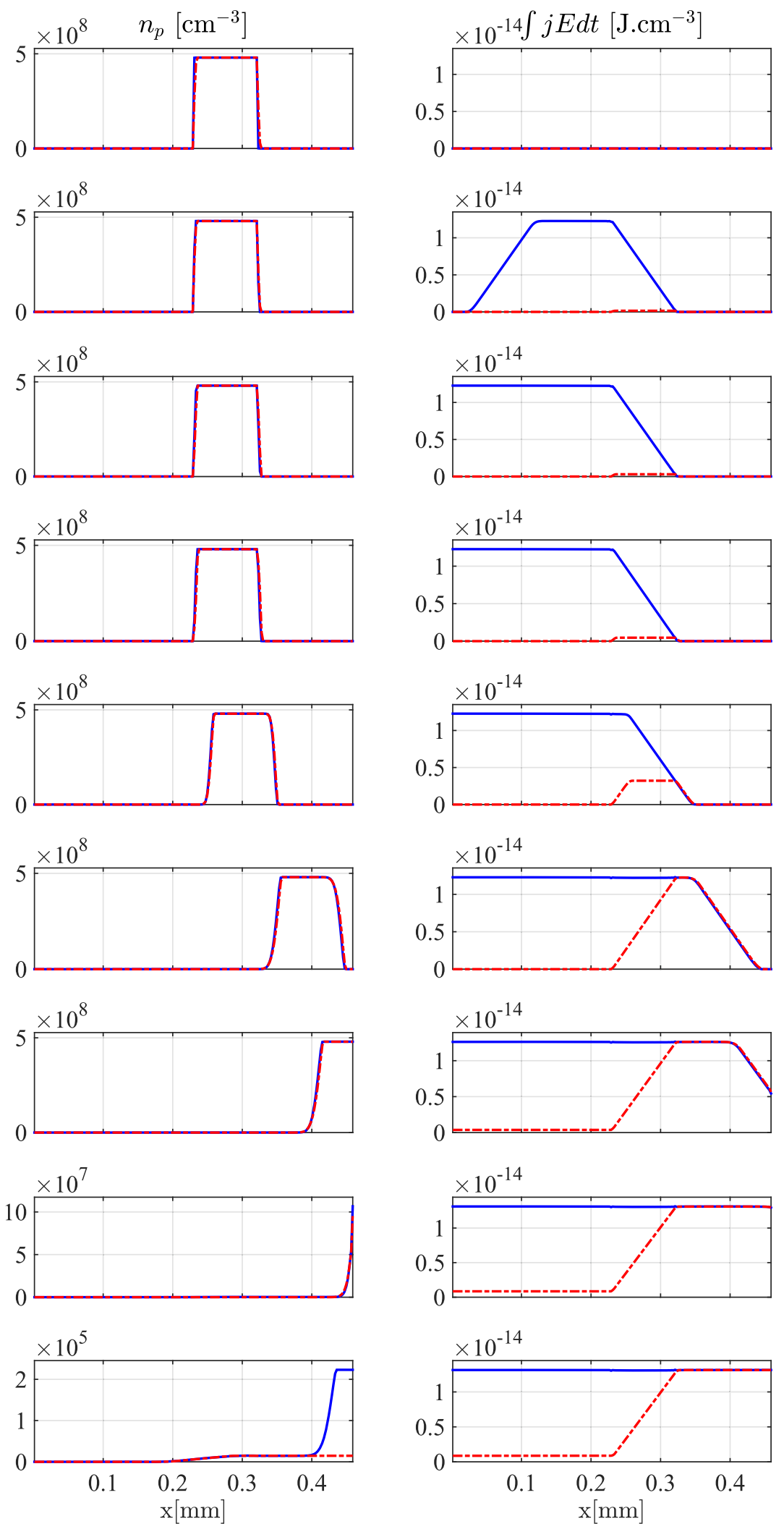

Figure 1. From left to right: spatial profiles of electron number density $n_{e}$, ion number density $n_{+}$and deposited electrical energy density for the $1 \mathrm{D}$ test case in nitrogen at standard temperature and pressure. The rows from top to bottom show the times $0,3,6,10,100,500,750$, 1000 and $2000 \mathrm{~ns}$. The results of the complete drift-diffusion-reaction model are drawn in blue, and those of the reduced model in red. 
plateau that extends until about the middle of the system, must have been created when the initial ion pulse hit the cathode, during the time interval of $600 \mathrm{~ns}$ to $1 \mu \mathrm{s}$. The cycle of arriving ion packages of decreasing amplitude continues until the gap is devoid of ions and the discharge decays.

The reduced model on the ion time scale is indicated in red. The first observation is that the ion motion of the reduced model agrees with the one of the full model for almost all times. But there are important differences in the electron density and the deposited energy:

1. Initially up to time $t=10 \mathrm{~ns}$, the electron density does not move out of the system, but disappears instantaneously in the first numerical time step. This is because the electron dynamics is not followed, but the electron density is determined by the electron density on the cathode together with the instantaneous equation (19a). An immediate consequence is that these electrons do not deposite energy in the system during the initial stage, and this lack of energy is seen very clearly until the last time step of the simulation. This difference has an impact on the solution, if the gas dynamics due to heating couples back onto the discharge model.

2. At later times, the electron density is approximated very well by the reduced model, as zooming into a narrow range of values of the electron density at times $t=750$ ns and $t=2 \mu \mathrm{s}$ shows. However, there is a major discrepancy at time $t=1 \mu \mathrm{s}$. This is because at this time the ion density on the cathode and therefore the rate of electron injection into the system changes so rapidly, that the adiabatic elimination of the electron dynamics is not valid anymore.

3. At time $t=2 \mu \mathrm{s}$, the ion density approximated by the reduced and the full model differ significantly close to the cathode. The staircase structure predicted by the full model in the region $x \in(0.4,0.48 \mathrm{~mm})$ represents the ions that were created by the initial seed of electrons via impact ionization while drifting out of the system. However, in the reduced model the initial seed of electrons did not create any ions in the gap (see point 1 above).

\subsection{Computational adaptivity to electron or ion time scale}

The observations above show that most of the time, the reduced model on the ion time scale approximates the full dynamics very well. Only during the time interval of the initial $8 \mathrm{~ns}$, and during a short time interval around $1 \mu \mathrm{s}$, the full dynamics differs from the reduced one. With an appropriate criterion the calculations can switch between the full and the reduced model and save substantial computing time.

We recall that in the reduced model the temporal derivative of the electron density $\partial_{t} n_{e}$ in $(2 a)$ and the space charge effects of the electron density in $(2 c)$ are neglected. In the present paper, the models are switched by hand, but we here briefly discuss possible programmed criteria that would allow the model to be adaptive in time.
For switching from the full to the reduced model, the size of $\partial_{t} n_{e}$ and $n_{e}$ relative to the other terms in equations (2a) and (2c) can be taken as a criterion. For switching from the reduced to the full model, $\partial_{t} n_{e}$ is not available, but the effective change of $n_{e}$ (due to changing distributions of ions, fields or gas density) within a numerical time step can be taken as a criterion. We will test the concept and develop a numerical algorithm for adaptivity in time in future work.

\section{Simulation and results}

\subsection{Set-up of the model}

We now present simulations of the evolution of an electric discharge in air at a pressure of $1 \mathrm{bar}$ and a temperature of $300 \mathrm{~K}$. The gap size of $1.38 \mathrm{~mm}$, and the applied voltage $3.7 \mathrm{kV}$, which amounts to an electric field of $27 \mathrm{kV} \mathrm{cm}^{-1}$ as long as space charge effects of the discharge can be neglected. In such as field below breakdown, an electrical discharge can develop only due to field enhancement at the tip of a streamer discharge [19] or due to a (local) decrease of air density. In the simulations presented here, the decreasing air density drives the discharge evolution.

On the cathode, secondary emission $(4 a)$ is included with a factor of $\gamma=0.3$ which means that 10 positive ions impacting on the cathode liberate 3 electrons on average from the surface into the discharge. A self-sustained Townsend discharge can develop and grow in a homogeneous field $E$ in a gas density $N$, if $\alpha_{\text {eff }}(E / N) \geqslant 1060 / \mathrm{m}$, according to (1).

To start the simulation, we place a seed of positive ions and electrons with a gaussian distribution given by

$$
\left.n(r, z)\right|_{t=0}=n_{0} \exp \left[-\frac{r^{2}}{\sigma_{r}^{2}}-\frac{\left(z-z_{0}\right)^{2}}{\sigma_{z}^{2}}\right],
$$

where we assign $n_{0}=6.9 \times 10^{16} \mathrm{~cm}^{-3}, z_{0}=0.69 \mathrm{~mm}$ and $\sigma_{r}=\sigma_{z}=23 \mu \mathrm{m}$. This amounts to approximately $2.2 \times 10^{9}$ electrons and ions. Since we carry out our simulations in air, the ratio of the initial number density of positive nitrogen ions to the initial number density of positive oxygen ions is taken as $4: 1$. The initial velocity $\mathbf{v}$ of the background gas is set equal to zero.

We perform the simulation in 3D with cylindrical symmetry around the $z$-axis (effectively 2D in $r, z$ coordinates). In the radial direction the domain extends from $r=0 \mathrm{~mm}$ to $r=4.14 \mathrm{~mm}$. The ionic species included are $\mathrm{N}_{2}^{+}, \mathrm{O}_{2}^{+}$and $\mathrm{O}_{2}^{-}$. As in section 3, we neglect electron diffusion in the reduced model. However, ion diffusion is taken into account in both the full model and the reduced model. The transport and reaction coefficients were read from a data file which was generated from online calculation tool BOLSIG+ (dated 27-06-2016) with the Phelps database $[20,21]$ from the LXCat website. The applied field is slightly below the breakdown value of $27.5 \mathrm{kV} \mathrm{cm}^{-1}$ at standard temperature and pressure, and therefore the effective ionization coefficient $\alpha_{\text {eff }}$ is negative. In the current work, we have not considered detachment processes. It will be included in our future work where chemical pathways will be treated more comprehensively. 
For details regarding the numerical implementation of the schemes used in the computation of physical quantities related to electrical discharge and gas, we refer the reader to appendix.

We switch from the full model to the reduced model at time $t=10 \mathrm{~ns}$ to accelerate the computation. At this moment of time the primary electrons have left the system and the system comprises of heavier ionic species. Furthermore, the contribution to the space charge is dominated by the ionic species and therefore the electric field profile is well approximated by the ionic distribution.

\subsection{Simulation results}

Figures 2 and 3 show the time evolution of electron density $n_{e}$, density $n_{p}$ of positive oxygen and nitrogen ions, density $n_{\mathrm{O}_{2}^{-}}$of negative oxygen ions, effective Townsend coefficient $\alpha_{\text {eff }}(E / N)$, air temperature $T$ and ratio $\rho / \rho_{0}$ of air density $\rho$ over air density $\rho_{0}$ at standard temperature and pressure. In figure 4 , we plot the temporal evolution of charge densities, gas temperature, gas pressure and relative gas density at the points $z=0.3 L_{z}$ (lower-half) and $z=0.7 L_{z}$ (upper-half) at time steps $t=0,0.29,0.58,0.86,1.15,1.44,1.72$, $2.01,2.29,2.58,2.86,3.15,4.29,11.4$ and $25 \mu \mathrm{s}$. As the system evolves, we first observe activity in the upper-half due to the relatively fast movement of electrons. On the longer timescale, activity in the lower half is observed when the ions move. Figures 2 and 3 show the full gap between the electrodes, but they zoom in into the structures near the center and do not show the full radial extension of the simulation. The temporal evolution is organized in three groups, where each group uses the same color scheme for densities and fields.

The figures show the following distinct stages of evolution.

\subsubsection{Drift of the initial electrons to the anode up to time $\approx 7$} ns. The simulation starts with a concentrated ionization seed with an equal number of electrons and positive ions, while there are no negative ions in the system. Electric field, temperature and air density are constant in space. The Townsend coefficient $\alpha_{\text {eff }}$ is constant and negative.

Up to approximately $7 \mathrm{~ns}$, the electrons drift upwards towards the anode and leave the system, while the positive ions are essentially immobile. While the electrons are drifting, their number decreases due to attachment and increases due to impact ionization; whether this relates to a net loss or a net gain, depends on the sign of $\alpha_{\text {eff }}$. The electron attachment can directly be seen in the form of negative ions left behind along the electron trail. Due to the high value of the reduced electric field $E / N$, the attachment is quite strong and hence the number of negative oxygen ions quite large.

The electrons also generate Ohmic heat along their trail. This causes the local temperature $T$ to rise up to a maximum of $500 \mathrm{~K}$ after $10 \mathrm{~ns}$. As we assume that only $15 \%$ of the Ohmic heat is released directly, while $85 \%$ is stored in vibrational states and released as heat on a time scale of $20 \mu \mathrm{s}$, the local temperature increases further up to a maximum of $720 \mathrm{~K}$ at time $293 \mathrm{~ns}$. On the other hand, the time is too short for thermal expansion of the air, so the air density $N$ or $\rho$ changes by approximately $1 \%$. The assumption of $\eta(E / N)=15 \%$ is based on previous work [3, 22]. Furthermore, in this work the ultrafast VT-relaxation timescale of the vibrationally excited $\mathrm{N}_{2}(\nu)$ is treated as a parameter. We chose its value to be $20 \mu \mathrm{s}$. It was a choice based on work by Komuro et al [23]

The effective Townsend coefficient $\alpha_{\text {eff }}$ depends on the reduced electric field $E / N$; it is mostly negative, but reaches positive values above the electron package and below the ion package. As air density $N$ has hardly changed, this indicates a local enhancement of the field $E$ due to the local space charges.

4.2.2. Switching from full to reduced model at time $10 \mathrm{~ns}$. The numerical test of the last section has shown that the electrons have to be traced with the full dynamics during the initial stage. Otherwise no heat would be deposited during this stage, and also the trail of negative ions would be missing. After the initial electrons have left the system, we switch to the reduced model at time $t=10 \mathrm{~ns}$ for the rest of the simulation.

We mention that in previous work [16] we have started right away with the reduced model in a study of electric breakdown in supercritical nitrogen and therefore missed the heat deposited during the initial stage and its effects on the further dynamics.

\subsubsection{Drift of the initial ions to the cathode up to time $1.7 \mu \mathrm{s}$.}

The positive ions are initially inserted in the middle of the gap. In the initial air density and electric field they would reach the electrode after approximately $1.7 \mu \mathrm{s}$. And this is what they do, despite some field enhancement and decrease of air density. The trail of negative ions moves upwards towards the anode within the same time. As there is no source of electrons, the electron density vanishes after the initial electrons have reached the anode.

While the ions move, they deposit Ohmic heat. In the time frame $t=0-293 \mathrm{~ns}$ the heat deposited in the upper part of the system is significantly greater than in the lower part of the system. This occurs because in that time frame the ions have not drifted much and hence have not deposited substantial heat. In comparison, the electrons move rapidly and deposit electrical energy as heat while creating more electrons (and ions) on their way out. On the longer timescale, the ions start drifting and deposit heat in the lower part of the system. This can be observed in the gas temperature profile on longer timescale where the temperature rises as the ions drift downward. Furthermore, the density of the postive ions also decreases due to radial diffusion as they drift downward.

The increased air temperature drives an expansion wave; hence the air density $\rho$ decreases by up to $3 \%$ in the center and increases by up to $2 \%$ in the expansion shock wave. The change of $N$ or $\rho=m_{w} N$ also leads to a change of the effective Townsend coefficient $\alpha_{\text {eff }}$. 

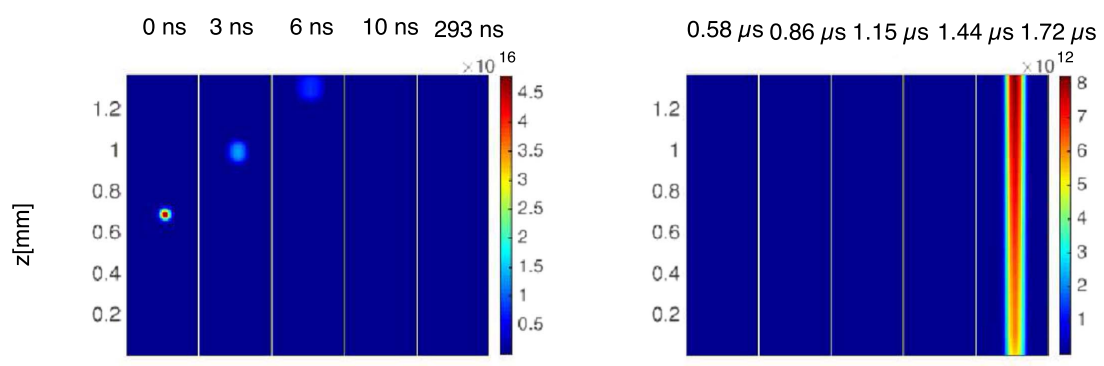

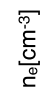
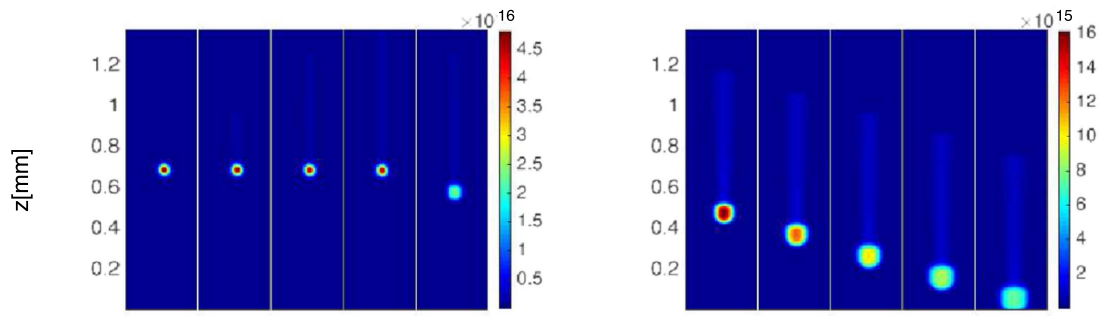

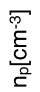
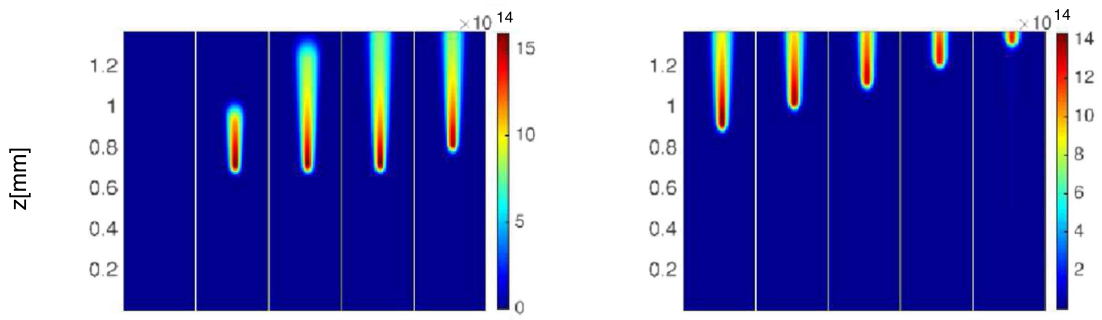

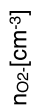
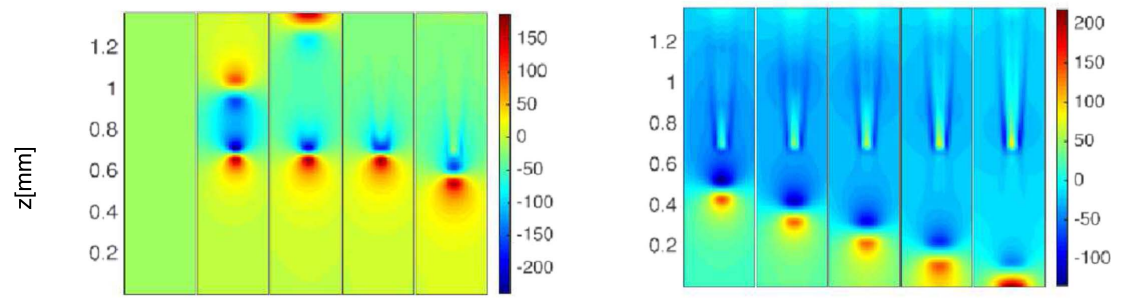

\section{$\underset{\frac{F}{E}}{\frac{F}{\delta}}$}
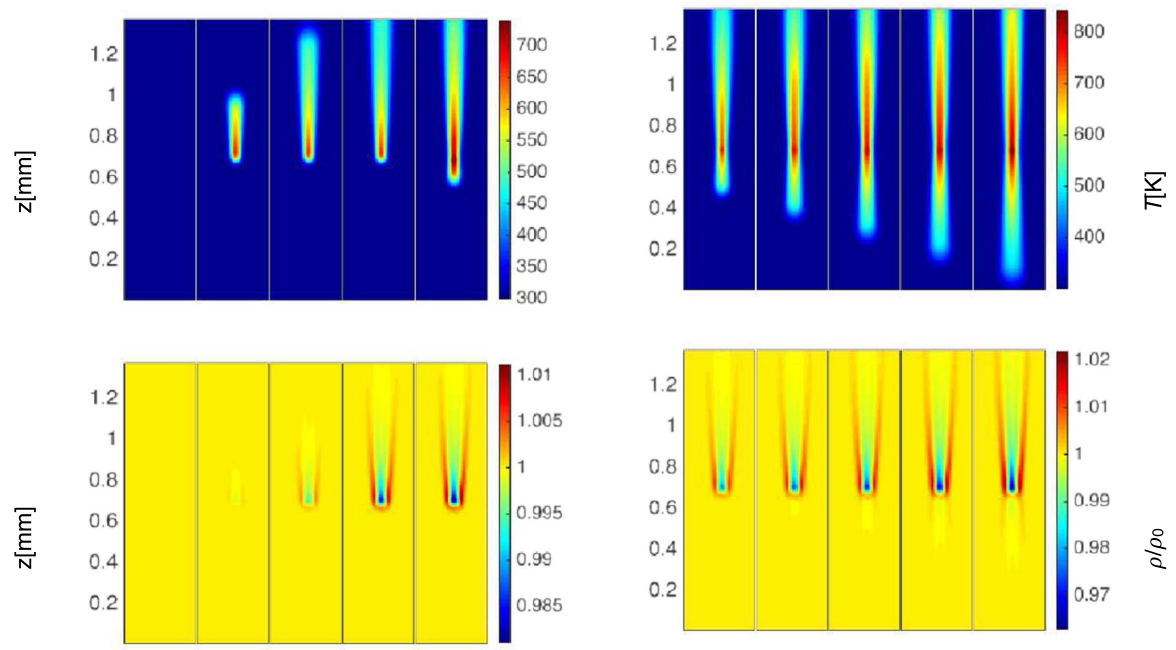

Figure 2. Evolution of electron number density $n_{e}$, positive ion number density $n_{p}$, negative ion number density $n_{\mathrm{O}_{2}^{-}}$, effective Townsend coefficient $\alpha_{\text {eff }}$, gas temperature $T$ and normalized gas density $\rho / \rho_{0}$. The initial seed of electrons and positive ions is placed on the discharge axis $(r=0)$ at $z=0.69 \mathrm{~mm}$. The gas is air at 1 bar and a voltage of $3.7 \mathrm{kV}$ is applied to a gap of $1.38 \mathrm{~mm}$. The temporal sequence for $t=0$, $3,6,10$ and $293 \mathrm{~ns}$ in the left panel are plotted with one color scheme for densities and fields, and the sequence for $t=580$ and $867 \mathrm{~ns}$, and $1.15,1.44,1.72 \mu \mathrm{s}$ in the right panel with another color scheme. The full height of the system is shown, but the plots are truncated in the radial direction. 

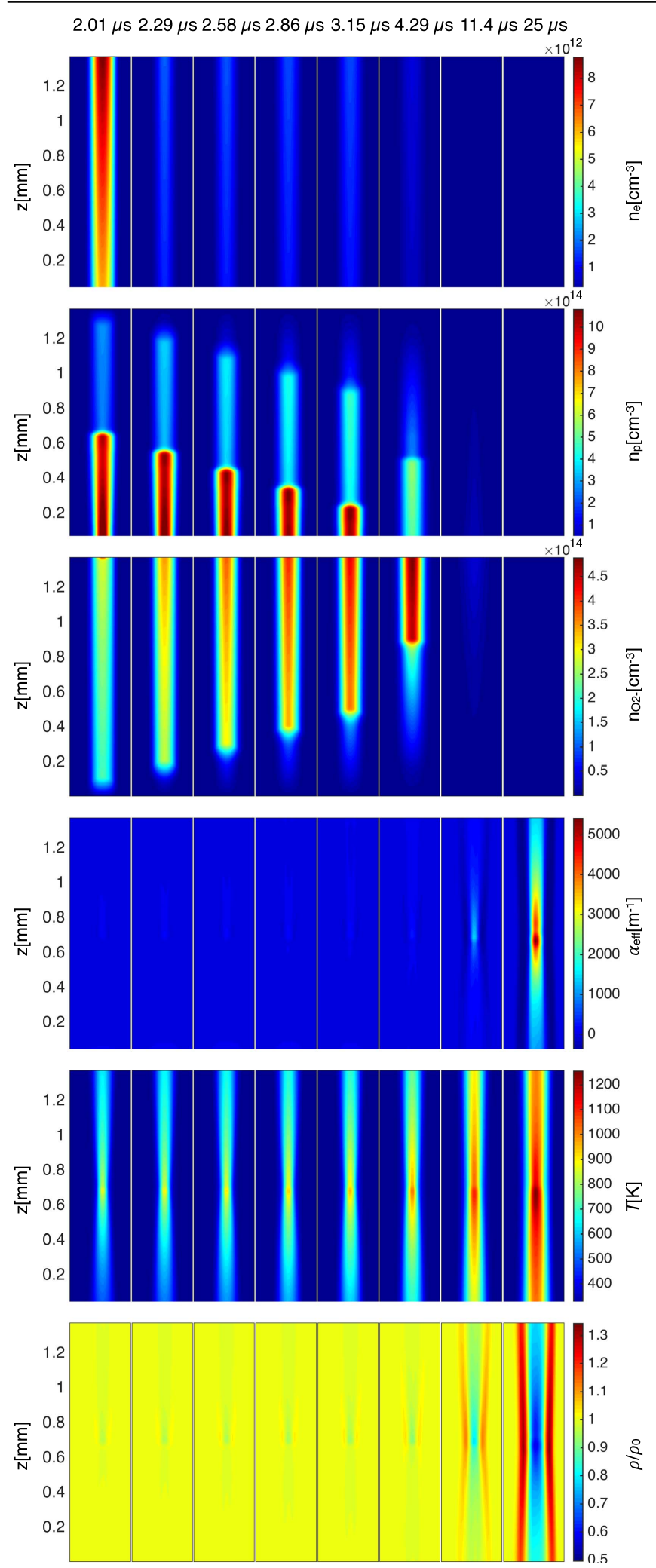

Figure 3. Continuation of the previous figure with plots for $t=2.01$, $2.29,2.58,2.86,3.15,4.29,11.4$ and $25 \mu$ s, again with a different color scheme for the densities and fields.

4.2.4. Second and third ionization wave and electric breakdown due to heating. At time $t=1.72 \mu \mathrm{s}$, the positive ions reach the cathode and liberate electrons through secondary emission. These electrons on their way up to the anode create ionization along their trail. In the plot at time $1.72 \mu \mathrm{s}$, this can only be seen in the electron density. But at time $2.01 \mu \mathrm{s}$, when the primary ions have left the system, the color coding of the ion density in the figure is changed, and two structures of positive ion columns are visible. The 'blue' column (corresponding to a density of $\approx 2 \times 10^{14} / \mathrm{cm}^{3}$ ) over almost the whole height of the system is the positive ion density created at that instance by the electrons. The negative ion density shows a corresponding column due to electron attachment along their trail. The 'red' column (corresponding to a density of positive ions of $\approx 1 \times 10^{15} / \mathrm{cm}^{3}$ ) in the lower half of the plot is the ion density created while the primary electrons drifted out of the system. This column in the mean time has drifted down by half the gap length.

Each column of positive ions will create another ionization wave when reaching the cathode, so the cycle repeats, but the amplitude decreases because the field is below breakdown, similarly to the $1 \mathrm{D}$ case discussed in the previous section. The difference lies in the fact that now air heating and expansion is included. The temperature keeps increasing in the channel and drives an expansion shock wave where the air density increases by $30 \%$, while it decreases in the channel by $50 \%$ at time $25 \mu \mathrm{s}$. This leads to a large increase of the reduced electric field $E / N$ and of the ionization rate and paves the way to full electric breakdown. In fact, already at time $t=11.4 \mu \mathrm{s}$, the minimum value of $\alpha_{\text {eff }}$ along the discharge axis is larger than $1060 \mathrm{~m}^{-1}$ which suffices to support a Townsend discharge according to equation (1). Figure 5 shows the temporal evolution for the total input energy deposited on the axis of the discharge. A fraction $\eta=0.15$ of the input energy instantaneouly heats the gas and the remaining energy is relaxed with a VT-relaxation timescale of $20 \mu \mathrm{s}$.

The simulation can be continued until time $t=35 \mu \mathrm{s}$ when temperature reaches a maximum of about $20000 \mathrm{~K}$, but then our physical model with a majority of non-ionized air molecules is certainly no longer applicable.

We also carried out simulation (not included in this work) with $\eta=0.3, \tau=100$ ns and reduced initial seed size. We found the results to be qualitatively similar to the results obtained above. Since the VT relaxation timescale of $100 \mathrm{~ns}$ is much smaller as compared to $20 \mu \mathrm{s}$, the heating takes place at a faster rate resulting is an earlier breakdown.

\section{Summary and outlook}

We have simulated the dynamics of a coupled system of electric discharge and background gas in air at standard temperature and pressure in a short gap in plane-to-plane electrode configuration, assuming cylindrical symmetry. Our discharge model includes electron impact ionization, attachment and secondary emission in fluid approximation with coefficients depending on electric field and gas density, and the hydrodynamic model for the carrier gas accounts for Ohmic heating and the associated gas expansion. 

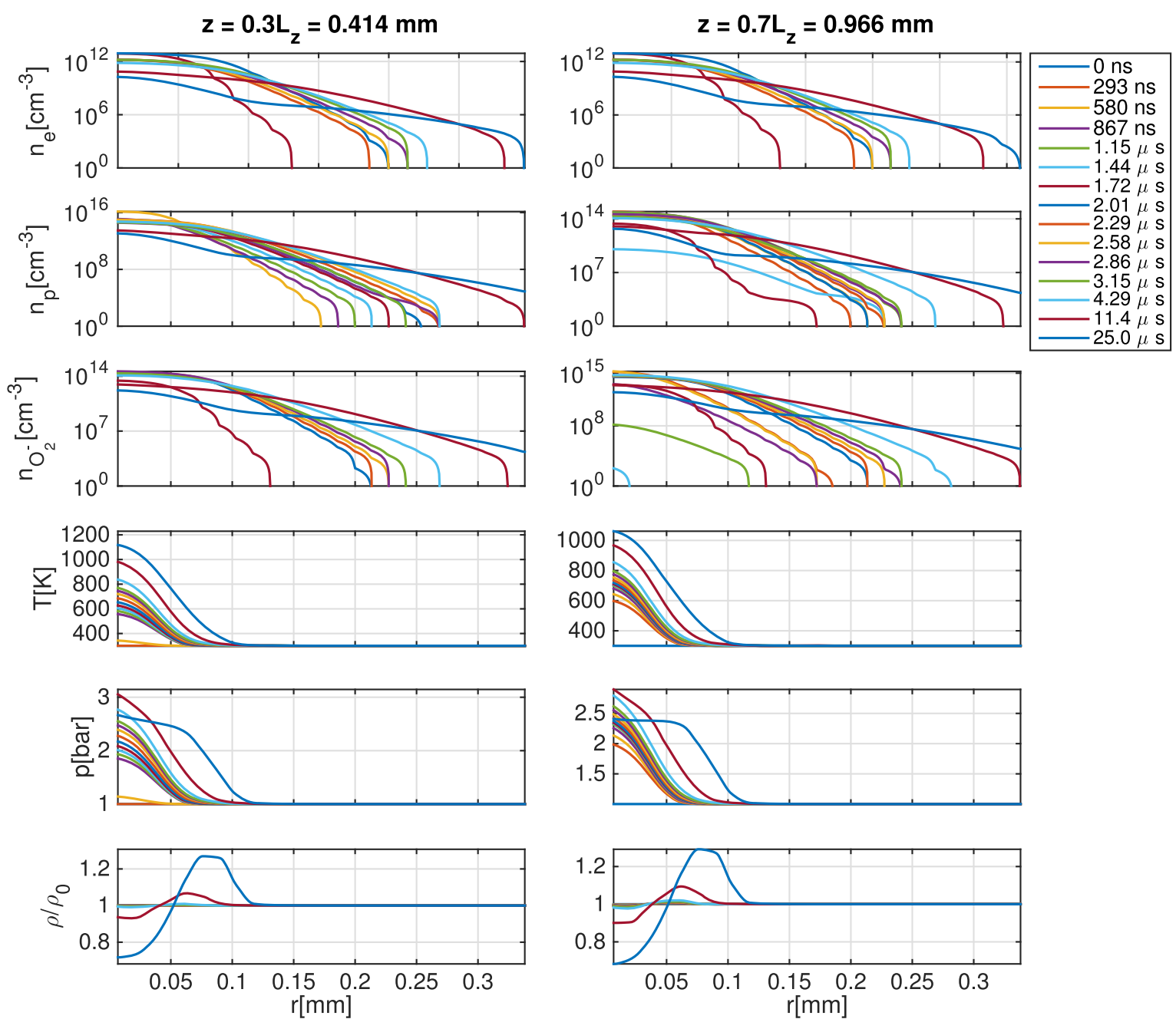

Figure 4. Temporal evolution for the charged particle densities, gas temperature, gas pressure and relative gas density at $z=0.3 L_{z}=0.414 \mathrm{~mm}$ and $z=0.7 L_{z}=0.966 \mathrm{~mm}$. Plotted are time steps $t=0,0.29,0.58,0.86,1.15,1.44,1.72,2.01,2.29,2.58,2.86$, $3.15,4.29,11.4$ and $25 \mu \mathrm{s}$.

To treat the widely varying time scales of electron and ion dynamics, gas heating and expansion, we have derived a reduced model on the (slow) time scale of ion motion. In previous work [16], we had used the reduced model only. In the present paper, we show that this approach leads to missing some heat deposition during the initial stage, and to errors at well defined later stages. We therefore now switch between the full model on the (fast) electron timescale and the reduced model on the (slow) ion timescale. This adaptivity in time largely enhances computational efficiency.

Our simulations show how the air gap eventually breaks down due to gas heating, rather than due to the streamer mechanism which is driven by space charge effects. We observe a cyclic process: positive ions hit the cathode, liberate electrons via secondary emission, and these electrons feed the discharge channel by producing more electrons and ions via impact ionization. The newly created ions again drift toward the cathode. The moving electrons and ions heat the gas, the gas expands, and eventually electric breakdown occurs in an unchanged electric field due to the decreased gas density near the discharge axis.

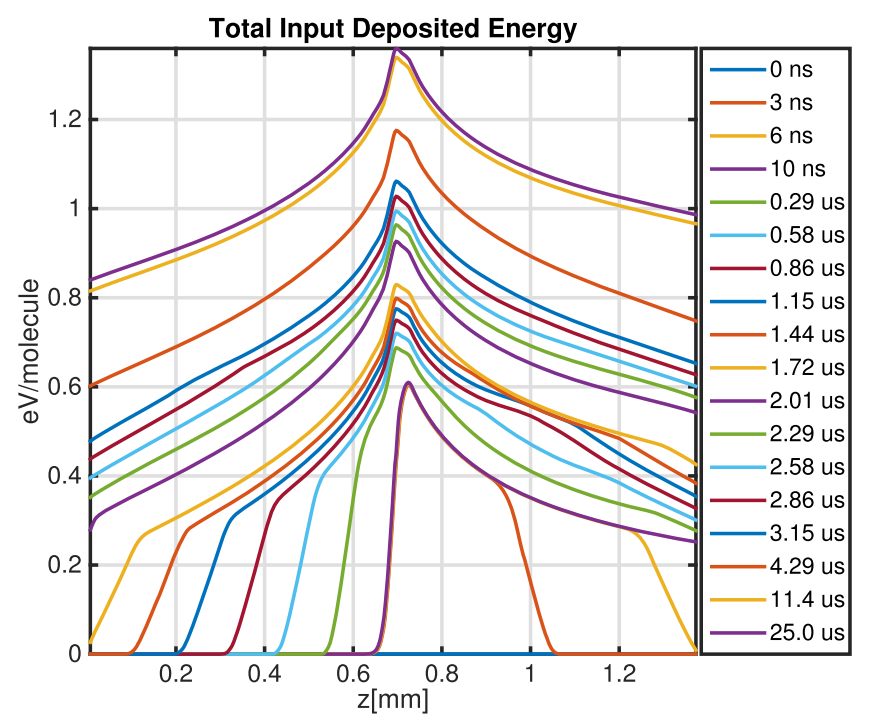

Figure 5. Temporal evolution for the total input energy deposited on the axis of the discharge. A fraction $\eta=0.15$ of the input energy instantaneouly heats the gas and the remaining energy is relaxed with a VT-relaxation timescale of $20 \mu \mathrm{s}$. The time steps plotted are the same as in figures 2 and 3. 
While in the present work we switched by hand from the full to the reduced model, we will study the switching criteria in more detail in future work to provide automatic time adaptivity of the computational code. We also intend to include more chemical and ion species in future 2D modeling to better understand the combined contributions of gas expansion and changing gas composition.

\section{Acknowledgments}

AA acknowledges the financial support under the Industrial Partnership Programme 'Computational Sciences for Energy Research' (CSER) of the Foundation for Fundamental Research on Matter (FOM), which is part of the Netherlands Organisation for Scientific Research (NWO).

\section{Appendix. Numerical implementation}

In this appendix, we discuss the numerical methods we employed to disctretize our equations. We recall that in this work we have used a strategy where we first carry out the simulation of the full model (drift-diffusion-reaction model for elefctric discharge) coupled to the Euler equations on the timescale of electron motion. After the primary electrons have left the system, we switch to the reduced model for electric discharge coupled to the Euler equations and carry out the simulation on the timescale of ion motion. Below we briefly describe the discretization schemes we employed to carry out the simulation in both these situations.

We adopt a finite volume approach for computation whereby we employ a conservative flux limited scheme $[24,25]$ with Koren flux limiter for discretization in space. These discretizations are similar to those by Montijn et al [26]. The model after discretization in space is a differential algebraic system. Formally, the system can be written as

$$
\begin{gathered}
d_{t} P=F(P, Q), \\
0=G(P, Q),
\end{gathered}
$$

where in the full model (A.1a) represents the system of coupled ODEs describing the electrons, ions and gas variables and (A.1b) describes the electric field. In the reduced model (A.1a) represents the system of coupled ODEs describing the ions and gas variables and (A.1b) describes the electrons and the electric field. Now the equation (A.1b) can be solved in terms of variable $P$ such that

$$
Q=H(P) \text {. }
$$

The details of actual implementation will be described below (appendix A.1). The resulting system of ODEs then is given by

$$
d_{t} P=\Psi(P) \text {, where } \Psi(P)=F(P, H(P)) .
$$

This is evolved in time using a second-order Runge-Kutta method (explicit trapezoidal rule) [27]. In terms of the original system (A.1), this can be described in two stages, the first of which reads

$$
P_{n+1}^{*}=P_{n}+\Delta t F\left(P_{n}, Q_{n}\right),
$$

$$
Q_{n+1}^{*}=H\left(P_{n+1}^{*}\right),
$$

where $P_{n}$ and $Q_{n}$ represent the value of the variables $P$ and $Q$ at time $t=t_{n}$. Finally, the value of the variables at time $t=t_{n+1}$ are

$P_{n+1}=P_{n}+\frac{1}{2} \Delta t F\left(P_{n}, Q_{n}\right)+\frac{1}{2} \Delta t F\left(P_{n+1}^{*}, Q_{n+1}^{*}\right)$,

$$
Q_{n+1}=H\left(P_{n+1}\right) .
$$

The time-step is chosen such that the CFL condition for numerical stability is satisfied.

\section{A.1. Computation of electron density in reduced model}

In the reduced model we compute the electron density that now is of the form (A.1b), as follows. For simplicity, we first discuss the strategy in 1D case and later give the generalized form in 2D. The equation describing the electron dynamics in the reduced model of electric discharge that we adopt (after comparison with the drift-diffusion-reaction model) is:

$$
\frac{\partial}{\partial x} j_{e}=S_{e}
$$

where the total current density $j_{e}=-n_{e} \mu_{e} E$ and source term $S_{e}=\alpha\left|j_{e}\right|$. We have set electron diffusion coefficient $D_{e}$ to zero in the total electron current density. For a given time $t$, the above equation is an ODE with $x$ as the independent variable. The initial condition is given by $j_{e}^{b}=\gamma j_{p}^{b}$, where $j_{p}^{b}$ is the ion current density evaluated at the cathode and $\gamma$ denotes the secondary emission coefficient. We can then adopt an ODE method (with $x$ serving as the independent variable) to compute the electron current density (and hence the electron number density $\left.n_{e}=\left|j_{e}\right| /\left(\mu_{e} E\right)\right)$ at the next step given the value at the previous step. Note that in the reduced model the contritbution of electrons to the space charge is negligible and the electric field in completely determined by the distribution of the ions. For discretization we use the extrapolated second-order BDF2 method applied to (A.6) in backward direction:

$$
\frac{3}{2} j_{e}^{m}-2 j_{e}^{m+1}+\frac{1}{2} j_{e}^{m+2}=-2 \Delta x S_{e}^{m+1}+\Delta x S_{e}^{m+2},
$$

where $\Delta x$ is the width of a grid cell and $m$ is the position index such that $m=M-2, M-3, \ldots, 3,2,1$, where $M$ is the number of grid cells in 1D. This two-step method needs $j_{e}^{M}$ and $j_{e}^{M-1}$ as starting values. To compute $j_{e}^{M}$ we use the Euler method,

$$
j_{e}^{M}=j_{e}^{b}-\frac{\Delta x}{2} S_{e}^{b},
$$

where $j_{e}^{b}$ and $S_{e}^{b}$ denote the electron current density and the source term for electrons evaluated at the cathode respectively. Furthermore, $j_{e}^{M-1}$ is computed by integrating equation (A.6) using a second-order Runge Kutta method 
(explicit trapezoidal rule) with $j_{e}^{M}$ as initial value and timestep equal to $\Delta x$ as in appendix A.1

The strategy described above can be generalized to 2Dcylindrically symmetric geometry. The equation describing the electron dynamics is:

$$
\frac{\partial}{\partial z} j_{e_{z}}+\frac{1}{r} \frac{\partial}{\partial r} j_{e_{r}}=S_{e},
$$

where $j_{e_{z}}=-n_{e} \mu_{e} E_{z}, j_{e_{r}}=-n_{e} \mu_{e} E_{r}$ and $S_{e}=\alpha \mu_{e}|\mathbf{E}| n_{e}$. The above equation can be written as:

$$
\frac{\partial}{\partial z} j_{e_{z}}=\bar{S}_{e}
$$

where $\bar{S}_{e}$ is the modified source term given by:

$$
\bar{S}_{e}=S_{e}-\frac{1}{r} \frac{\partial}{\partial r} j_{e_{r}} .
$$

The equation (A.10) can now be integrated in exactly the same way as in the $1 \mathrm{D}$-case with $z$ serving as the independent variable.

\section{A.2. Implementation of heating term in Euler system}

The source term describing Joule heating of the gas is according to equation (10)

$$
\begin{aligned}
S_{\xi}(\mathbf{r}, t)= & \eta P(\mathbf{r}, t)+(1-\eta) \\
& \times \int_{-\infty}^{t} P\left(\mathbf{r}, t^{\prime}\right) \mathrm{e}^{-\left(t-t^{\prime}\right) / \tau} \frac{\mathrm{d} t^{\prime}}{\tau},
\end{aligned}
$$

where $\tau$ denotes the relaxation time constant of the excited vibrational energy states of the gas. The calculation of the temporal integral can be simplified by noting that

$$
\begin{aligned}
& \mathcal{I}(\mathbf{r}, t+\Delta t):=\int_{-\infty}^{t+\Delta t} P\left(\mathbf{r}, t^{\prime}\right) \mathrm{e}^{-\left(t+\Delta t-t^{\prime}\right) / \tau} \frac{\mathrm{d} t^{\prime}}{\tau} \\
& =\mathrm{e}^{-\Delta t / \tau} \mathcal{I}(\mathbf{r}, t)+\int_{t}^{t+\Delta t} P\left(\mathbf{r}, t^{\prime}\right) \mathrm{e}^{\left(t+\Delta t-t^{\prime}\right) / \tau} \frac{\mathrm{d} t^{\prime}}{\tau},
\end{aligned}
$$

so we only have to perform the integral from time $t$ to $t+\Delta t$ to update the integral $\mathcal{I}$. The source term can be computed to desired accuracy by computing the integral terms via quadrature rules; we have used the simple Trapezoidal rule.

\section{A.3. Implementation of outflow boundary condition in the Euler system}

To implement the outflow boundary condition in the Euler system on the outer radial boundary $\left(r=L_{r}\right)$ we extrapolate $\rho, v_{r}$ and $p$. For that we fit a second-order polynomial for the variables $\rho, v_{r}$ and $p$ through the points $\left(r_{M-2}, z_{n}\right),\left(r_{M-1}, z_{n}\right)$ and $\left(r_{M}, z_{n}\right)$, where the index $M$ refers to the outermost cellcenters in the radial direction and $n$ refers to index in the $z$ direction. Thereafter, we compute the value of the variable $\left(\rho, v_{r}\right.$ and $\left.p\right)$ by evaluating the respective polynomial on the outer radial boundary.

\section{References}

[1] Popov N 2001 Investigation of the mechanism for rapid heating of nitrogen and air in gas discharges Plasma Phys. Rep. 27 886-96

[2] Aleksandrov N, Kindysheva S, Nudnova M and Starikovskiy A Y 2010 Mechanism of ultra-fast heating in a non-equilibrium weakly ionized air discharge plasma in high electric fields J. Phys. D: Appl. Phys. $\mathbf{4 3}$ 255201

[3] Popov N 2011 Fast gas heating in a nitrogen-oxygen discharge plasma: I. Kinetic mechanism J. Phys. D: Appl. Phys. 44 285201

[4] Rusterholtz D, Pai D, Stancu G, Lacoste D and Laux C 2012 Ultrafast heating in nanosecond discharges in atmospheric pressure air 50th AIAA Aerospace Sciences Meeting vol 509 (Nashville, Tennessee, 9-12 January, 2012) AIAA

[5] Guerra V, Sá P and Loureiro J 2004 Kinetic modeling of lowpressure nitrogen discharges and post-discharges Eur. Phys. J. Appl. Phys. 28 125-52

[6] Starikovskaia S 2006 Plasma assisted ignition and combustion J. Phys. D: Appl. Phys. 39 R265

[7] Riousset J A, Pasko V P and Bourdon A 2010 Air-densitydependent model for analysis of air heating associated with streamers, leaders, and transient luminous events J. Geophys. Res.: Space Phys. 115 A12

[8] Marode E, Bastien F and Bakker M 1979 A model of the streamer-induced spark formation based on neutral dynamics J. Appl. Phys. 50 140-6

[9] Bastien F and Marode E 1985 Breakdown simulation of electronegative gases in non-uniform field J. Phys. D: Appl. Phys. 18377

[10] Shneider M 2006 Turbulent decay of after-spark channels Phys. Plasmas 13073501

[11] Komuro A and Ono R 2014 Two-dimensional simulation of fast gas heating in an atmospheric pressure streamer discharge and humidity effects J. Phys. D: Appl. Phys. 47 155202

[12] Tholin F and Bourdon A 2013 Simulation of the hydrodynamic expansion following a nanosecond pulsed spark discharge in air at atmospheric pressure J. Phys. D: Appl. Phys. 46 365205

[13] Kacem S, Ducasse O, Eichwald O, Yousfi M, Meziane M, Sarrette J P and Charrada K 2013 Simulation of expansion of thermal shock and pressure waves inducaed by a streamer dynamics in positive de corona discharges IEEE Trans. Plasma Sci. 41 942-7

[14] Šijačić D D, Ebert U and Rafatov I 2005 Oscillations in dc driven barrier discharges: numerical solutions, stability analysis, and phase diagram Phys. Rev. E 71 066402

[15] Rafatov I R, Šijačić D D and Ebert U 2007 Spatiotemporal patterns in a dc semiconductor-gas-discharge system: stability analysis and full numerical solutions Phys. Rev. E 76036206

[16] Agnihotri A, Hundsdorfer W and Ebert U 2016 Coupling discharge and gas dynamics in streamer-less spark formation in supercritical $\mathrm{N}_{2}$ Japan. J. Appl. Phys. 55 07LD06

[17] Kulikovsky A 1997 Positive streamer between parallel plate electrodes in atmospheric pressure air J. Phys. D: Appl. Phys. 30441

[18] Montijn C and Ebert U 2006 Diffusion correction to the raether-meek criterion for the avalanche-to-streamer transition J. Phys. D: Appl. Phys. 392979

[19] Sun A, Teunissen J and Ebert U 2014 The inception of pulsed discharges in air: simulations in background fields above and below breakdown J. Phys. D: Appl. Phys. 47445205 
[20] Phelps A V and Pitchford L C 1985 Anisotropic scattering of electrons by $\mathrm{N}_{2}$ and its effect on electron transport Phys. Rev. A 31 2932-49

[21] Lawton S and Phelps A 1978 Excitation of the $b^{1} \Sigma_{g}^{+}$state of $\mathrm{O}_{2}$ by low energy electrons J. Chem. Phys. 69 1055-68

[22] Ono R, Teramoto Y and Oda T 2010 Gas density in a pulsed positive streamer measured using laser shadowgraph J. Phys. D: Appl. Phys. 43345203

[23] Komuro A, Takahashi K and Ando A 2015 Numerical simulation for the production of chemically active species in primary and secondary streamers in atmospheric-pressure dry air J. Phys. D: Appl. Phys. 48215203
[24] Kurganov A and Tadmor E 2000 New high-resolution central schemes for nonlinear conservation laws and convection-diffusion equations J. Comput. Phys. 160 241-82

[25] Nessyahu H and Tadmor E 1990 Non-oscillatory central differencing for hyperbolic conservation laws J. Comput. Phys. 87 408-63

[26] Montijn C, Hundsdorfer W and Ebert U 2006 An adaptive grid refinement strategy for the simulation of negative streamers J. Comput. Phys. 219 801-35

[27] Hundsdorfer W and Verwer J G 2003 Numerical Solution of Time-Dependent Advection-Diffusion-Reaction equations vol 33 (Berlin: Springer) 